Hong Kong, dix ans après la rétrocession

\title{
Le mouvement pour la démocratie à Hong Kong Une décennie perdue?
}

Joseph Y.S. Cheng

\section{(2) OpenEdition}

Édition électronique

URL : http://journals.openedition.org/perspectiveschinoises/2723

ISSN : 1996-4609

Éditeur

Centre d'étude français sur la Chine contemporaine

Édition imprimée

Date de publication : 15 juillet 2007

ISSN : 1021-9013

\section{Référence électronique}

Joseph Y.S. Cheng, «Le mouvement pour la démocratie à Hong Kong Une décennie perdue ?»,

Perspectives chinoises [En ligne], 2007/2 | 2007, mis en ligne le 03 avril 2008, consulté le 02 mai 2019. URL : http://journals.openedition.org/perspectiveschinoises/2723

\section{(c) Tous droits réservés}




\title{
Le mouvement pour la
}

\section{démocratie à Hong Kong}

\author{
Une décennie perdue?
}

IOSEPH Y. S. CHENG

En 2007, le mouvement pour la démocratie à Hong Kong n'est pas au mieux de sa forme. Le suffrage universel ne sera sans doute pas instauré, même à l'échéance de 2012. La pérennité du mouvement est toutefois assurée par deux éléments : d'une part, toutes les parties concernées ont conscience de la nécessité de maintenir l'État de droit et la liberté d'information, d'autre part, une majorité d'électeurs hongkongais souhaite conserver un minimum d'équilibre des pouvoirs.

$\mathrm{D}$ epuis la mise en place de la Région administrative spéciale de Hong Kong (RASHK) en 1997, il n'y a pas eu de dialogue formel avec les autorités chinoises. Les manifestations massives du $1^{\text {er }}$ juillet 2003 et la crise politique qui s'est ensuivie (1) n'ont fait qu' exacerber le sentiment d'insécurité de Pékin, qui, dès lors, n'a eu de cesse de limiter l'influence du mouvement démocratique. C'est ainsi que, lors d'élections aussi déterminantes que celles du Conseil législatif (Legco) en septembre 2004, les partis démocrates considèrent que leur adversaire n'était autre qu'une machine d'État dirigée par Pékin ${ }^{(2)}$.

Les Hongkongais attachent toujours la même importance au maintien de l'équilibre des pouvoirs. Voter pour le camp démocrate constitue pour eux un moyen de limiter le pouvoir du gouvernement de la RASHK. En 2007, alors même que la reconduction de Donald Tsang à la tête de l'exécutif semblait acquise et que les intentions de vote pour le candidat démocrate Alan Leong stagnaient aux alentours de 20\%, deux tiers des Hongkongais se déclaraient malgré tout en faveur d'un scrutin véritablement compétitif. Le mouvement démocrate ne s'attendait pas à une telle mobilisation en sa faveur lors des élections du Comité électoral en décembre $2006^{(3)}$.

Les valeurs des Hongkongais ont évolué. Depuis la rétrocession, les attentes en matière de croissance économique, de perspectives de carrière et d'augmentation des revenus ont probablement été revues à la baisse. Ils souhaitent que le gouvernement intervienne davantage dans la vie économique. $\mathrm{La}$ famille, la protection de l'environnement et des sites historiques de Hong Kong sont autant de nouvelles préoccupa- tions. $\mathrm{Ni}$ le gouvernement ni les grands partis politiques n'ont apparemment su appréhender ce changement de valeurs au sein d'une population qui, en retour, s'estime de moins en moins satisfaite de leurs résultats (voir tableaux 1 et 2).

Cet article se propose d'étudier le mouvement démocratique au cours de la décennie 1997-2007. Les attentes des Hongkongais, les dynamiques internes du mouvement, les changements de valeurs survenus au sein de la population, les difficultés rencontrées et les défis à relever seront autant d'angles d'analyse.

\section{Les attentes de la population}

D'après une étude de Thomas W.P. Wong, en 1997 les valeurs les plus importantes sélectionnées parmi quatre possibilités étaient pour les Hongkongais, dans l'ordre : la stabilité sociale ( $56 \%$ des personnes interrogées), la prospérité économique $(24,7 \%)$, la démocratie politique $(11,1 \%)$ et les libertés individuelles $(8,2 \%)^{(4)}$. Par temps de précarité politique, il était naturel de faire de la stabilité une priorité. Peut-être se rendaient-ls compte que, Hongkong étant une économie

1. Voir l'ouvrage de l'auteur, The July 1 Protest Rally: Interpreting a Historic Event, Hong Kong: City University of Hong Kong Press, 2005.

2. Voir l'article de l'auteur, "Hong Kong's Democrats Stumble," Journal of Democracy, vol. $16, n^{\circ} 1,2005$, pp. 138-152

3. Voir tous les grands quotidiens de Hong Kong parus les 11 et 12 décembre 2006.

4. Thomas W.P. Wong, "Core Values: Revelations from Research in Hong Kong's Social Indicators (1988-2001)," in Siu-kai Lau et al. (eds), Trends and Challenges in Social Development: Experiences of Hong Kong and Taiwan (in Chinese), Hong Kong: Institute of Asia-Pacific Studies, The Chinese University of Hong Kong, 2006, p. 108. 
Tableau 1. Satisfaction envers le gouvernement de la RASHK (Evaluation semi annuelle, 1997-2006)

\section{Question posée :}

êtes-vous satisfait de la performance globale du gouvernement de la RASHK ?

\begin{tabular}{|c|c|c|c|c|c|c|c|}
\hline Période & 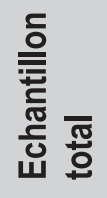 & $\begin{array}{c}\text { Très } \\
\text { satisfait }\end{array}$ & $\begin{array}{c}\text { Plutôt } \\
\text { satisfait }\end{array}$ & $\begin{array}{c}\mathrm{Ni} \\
\text { satisfait } \\
\text { ni } \\
\text { insatisfait }\end{array}$ & $\begin{array}{l}\text { Plutôt pas } \\
\text { satisfait }\end{array}$ & $\begin{array}{l}\text { Pas du tout } \\
\text { satisfait }\end{array}$ & $\begin{array}{l}\text { NSPI } \\
\text { Sans } \\
\text { opinion }\end{array}$ \\
\hline Juillet - Dec., 2006 & 6076 & $4,2 \%$ & $37,8 \%$ & $41,7 \%$ & $11,4 \%$ & $3,7 \%$ & $1,1 \%$ \\
\hline Jan. - Juin, 2006 & 7113 & $5,8 \%$ & $45,7 \%$ & $36,5 \%$ & $8,5 \%$ & $2,4 \%$ & $1,1 \%$ \\
\hline Juillet - Dec., 2005 & 6097 & $5,2 \%$ & $44,0 \%$ & $35,7 \%$ & $9,5 \%$ & $3,1 \%$ & $2,4 \%$ \\
\hline Jan. - Juin, 2005 & 6109 & $2,9 \%$ & $26,2 \%$ & $37,9 \%$ & $20,2 \%$ & $8,6 \%$ & $4,3 \%$ \\
\hline Juillet - Dec., 2004 & 6148 & $2,7 \%$ & $17,5 \%$ & $35,3 \%$ & $27,6 \%$ & $14,2 \%$ & $2,8 \%$ \\
\hline Jan. - Juin, 2004 & 6207 & $1,9 \%$ & $13,0 \%$ & $33,1 \%$ & $30,6 \%$ & $18,1 \%$ & $3,5 \%$ \\
\hline Juillet - Dec., 2003 & 7364 & $1,5 \%$ & $12,2 \%$ & $26,9 \%$ & $35,3 \%$ & $21,3 \%$ & $3,0 \%$ \\
\hline Jan. - Juin, 2003 & 6293 & $1,1 \%$ & $14,3 \%$ & $25,6 \%$ & $36,9 \%$ & $18,6 \%$ & $3,6 \%$ \\
\hline Juillet - Dec., 2002 & 6260 & $1,0 \%$ & $17,0 \%$ & $28,1 \%$ & $37,9 \%$ & $11,3 \%$ & $4,8 \%$ \\
\hline Jan. - Juin, 2002 & 6217 & $1,3 \%$ & $23,7 \%$ & $31,3 \%$ & $32,8 \%$ & $6,1 \%$ & $4,9 \%$ \\
\hline Juillet - Dec., 2001 & 6321 & $1,0 \%$ & $19,2 \%$ & $30,9 \%$ & $33,4 \%$ & $10,5 \%$ & $5,0 \%$ \\
\hline Jan. - Juin, 2001 & 6348 & $1,3 \%$ & $25,3 \%$ & $35,0 \%$ & $26,7 \%$ & $6,5 \%$ & $5,2 \%$ \\
\hline Juillet - Dec., 2000 & 6324 & $1,5 \%$ & $19,9 \%$ & $31,3 \%$ & $32,1 \%$ & $9,6 \%$ & $5,7 \%$ \\
\hline Jan. - Juin, 2000 & 4240 & $1,6 \%$ & $22,4 \%$ & $39,1 \%$ & $22,9 \%$ & $9,0 \%$ & $5,1 \%$ \\
\hline Juillet - Dec., 1999 & 3205 & $1,4 \%$ & $22,8 \%$ & $39,2 \%$ & $23,6 \%$ & $8,0 \%$ & $5,0 \%$ \\
\hline Jan. - Juin, 1999 & 4213 & $0,7 \%$ & $24,5 \%$ & $39,9 \%$ & $26,3 \%$ & $5,4 \%$ & $3,2 \%$ \\
\hline Juillet - Dec., 1998 & 4755 & $1,0 \%$ & $21,7 \%$ & $35,8 \%$ & $29,0 \%$ & $8,0 \%$ & $4,6 \%$ \\
\hline Jan. - Juin, 1998 & 3744 & $0,8 \%$ & $27,4 \%$ & $38,7 \%$ & $23,0 \%$ & $4,6 \%$ & $5,5 \%$ \\
\hline Juillet - Dec., 1997 & 3181 & $1,9 \%$ & $40,7 \%$ & $32,7 \%$ & $13,6 \%$ & $1,9 \%$ & $9,3 \%$ \\
\hline
\end{tabular}

Source: Site internet du Public Opinion Programme de l'Université de Hong Kong, POP Polls: People's Satisfaction with the HKSAR Government - half-yearly average. (http://hkupop.hku.hk/english/popexpress/sargperf/sarg/halfyr/datatables.html, le 28 mars 2007).

mature, une croissance rapide ne semblait donc plus à l'ordre du jour. Mais qu'est ce qui constitue les fondements de la stabilité ? Il est probable que la perception des éléments constitutifs de la stabilité sociale change avec le contexte. Ainsi, quand l'économie était en berne et le taux de chômage très élevé, les inquiétudes portaient principalement sur l'économie et le marché de l'emploi (voir tableau 3). Dans les mois qui précédaient juillet 2003, le mécontentement portait sur le projet d'article $23^{(5)}$ et le bilan du gouvernement de Tung Chee-Hwa (voir tableau 1).

Tung Chee-Hwa déclara lors de la présentation des grands axes de sa politique ${ }^{\left({ }^{(6)}\right.}$ que son gouvernement serait amené à
5. L'Article 23 de la Loi fondamentale (constitution de Hong Kong) stipule que «La Région Administrative Spéciale de Hong Kong élabore elle-même des lois interdisant tout acte de trahison, de sécession, de sédition et de subversion à l'égard du gouvernement central [les autorités de Pékin], tout vol de secrets d'Etat, interdisant à toute organisation politique ou institution de l'étranger de mener des activités politiques à Hong Kong et interdisant à toute organisation politique ou institution de Hong Kong d'établir des liens avec des organisations politiques ou institutions de l'étranger ». Cet article a été inséré dans le projet de Loi fondamentale après les grandes manifestations organisées à Hong Kong pour protester contre le massacre de Tiananmen en 1989. Le gouvernement chinois craignait visiblement que ce type de manifestations se reproduise. Le gouvernement de Tung a été assez sage pour ne pas entamer ce processus législatif controversé lors de son premier mandat. Le projet d'Article 23 a finalement été soumis à une consultation publique en septembre 2002. Comme prévu, le projet fit craindre des mesures de répression contre les groupes de défense des droits de l'homme et contre la secte Falun Gong. Pour le camp démocrate, le projet constituait aussi une atteinte aux libertés civiles. Voir South China Morning Post, 25 décembre 2002.

6. Voir la première déclaration politique de Tung: http://www.policyaddress.gov.hk/ pa97/english/paindex.htm, consulté le 28 février 2007 
Tableau 2. Evaluation des grands mouvements politiques et syndicaux, 1998-2006

\section{Question posée :}

Evaluez votre degré de soutien à XXX sur une échelle de 0 à 100,

0 indiquant un degré de soutien nul,100 un soutien inconditionnel et 50 un soutien moyen.

\begin{tabular}{llllllllll}
\hline Moyenne annuelle & 1998 & $\mathbf{1 9 9 9}$ & $\mathbf{2 0 0 0}$ & $\mathbf{2 0 0 1}$ & $\mathbf{2 0 0 2}$ & $\mathbf{2 0 0 3}$ & $\mathbf{2 0 0 4}$ & $\mathbf{2 0 0 5}$ & $\mathbf{2 0 0 6}$ \\
\hline HKFTU & 57,2 & 55,6 & 56,6 & 57,8 & 57,9 & 52,0 & 50,8 & 52,1 & 53,3 \\
\hline $\begin{array}{l}\text { A45 Concern Group/ } \\
\text { Civic Party* }\end{array}$ & - & - & - & - & - & - & 53,4 & 53,8 & 51,8 \\
HKCTU & - & 52,2 & 54,2 & 56,7 & 56,7 & 52,8 & 52,3 & 51,3 & 51,2 \\
\hline ADPL & - & 48,6 & 50,8 & 53,8 & 55,3 & 50,1 & 49,7 & 48,6 & 49,8 \\
\hline DAB & 51,6 & 50,5 & 50,7 & 53,3 & 53,7 & 45,3 & 42,9 & 47,0 & 49,7 \\
\hline Liberal Party & 50,2 & 48,5 & 48,3 & 50,5 & 51,7 & 49,7 & 49,1 & 48,7 & 49,3 \\
\hline Democratic Party & 56,3 & 52,3 & 51,3 & 52,6 & 51,5 & 50,2 & 51,1 & 47,9 & 47,0 \\
\hline The Frontier & 53,7 & 50,0 & 50,4 & 50,8 & 50,9 & 48,0 & 47,1 & 45,8 & 45,6 \\
\hline HKASPDMC & - & 49,2 & 48,4 & 49,5 & 48,4 & 46,4 & 45,9 & 45,6 & 43,1 \\
\hline AFA & - & - & 37,5 & 39,8 & 39,0 & 36,8 & 37,6 & 36,6 & 35,8 \\
\hline
\end{tabular}

Notes : 1. * Le A45 Concern Group s'est dissout en mars 2006 pour former le Civic Party

2. HKFTU $=$ Hong Kong Federation of Trade Unions

HKCTU $=$ Hong Kong Confederation of Trade Unions

$A D P L=$ Hong Kong Association for Democracy and People's Livelihood

$\mathrm{DAB}=$ Democratic Alliance for the Betterment of Hong Kong (Depuis 2005 Democratic Alliance for the Betterment and Progress of Hong Kong)

HKASPDMC $=$ Hong Kong Alliance in Support of Patriotic Democratic Movements of China

AFA = April Fifth Action

Source: Moyennes annuelles calculées à partir des données disponibles sur le site internet du Public

Opinion Programme de l'Université de Hong Kong, POP Polls: Rating of Top Ten Political Groups.

(http://hkupop.hku.hk/english/popexpress/pgrating/topten1.html, 28 février 2007)

prendre davantage d'initiatives. Le déclin de la compétitivité internationale de Hong Kong était à l'évidence l'une de ses préoccupations majeures. En se fixant pour objectif la construction de 85000 logements par an, il souhaitait rendre le logement plus abordable pour les Hongkongais de la classe moyenne. Cependant, il estimait aussi que les prix élevés de l'immobilier exerçaient une pression à la hausse sur la structure des coûts, rendant les biens et services plus coûteux et moins compétitifs. Pour améliorer la compétitivité de Hong Kong sur le long terme, il a donc donné la priorité à l'éducation. L'accent mis sur les services aux personnes âgées témoigne d'une véritable préoccupation à l'égard d'une population vieillissante et d'une prise de conscience des attentes accrues de la population en matière de services sociaux.
La baisse marquée des performances économiques de Hong Kong depuis 1997 est indéniablement à l'origine de nombreux maux et d'un grand mécontentement au sein de la population. Le taux de croissance annuel moyen du PIB par habitant est passé de 4,5\% pour la période 1983-1997 à 1,9\% pour la période 1997-2001. Le plein emploi a été plus ou moins maintenu de 1985 jusqu'à mi-1997, le taux de chômage oscillant entre 1,3\% et 3,5\% seulement. Depuis la rétrocession de Hong Kong, le taux de chômage est passé de $2,1 \% \mathrm{mi}-1997$ à un pic record de $8,7 \%$ mi-2003 ${ }^{(7)}$.

Mais la perte de vitesse de l'économie est bien antérieure: le taux de croissance réel du PIB par habitant est

7. Sung Yun-wing, "Hong Kong Economy in Crisis," in Siu-kai Lau (éd.), The First Tung Chee-hwa Administration, Hong Kong, The Chinese University Press, 2002, p. 123. 
passé d'une moyenne annuelle de $5,2 \%$ dans les années 1980 à 3,5\% pour la période 1990-1996 tandis que la croissance du PIB par actif est passée de $4,7 \%$ à $3,3 \%{ }^{(8)}$. Une équipe d'économistes de la Chinese University of Hong Kong a observé que la productivité globale des facteurs dans le secteur manufacturier n'a cessé de décliner de 1984 à 1993, date à laquelle " la production manufacturière n'atteignait que $87 \%$ de son niveau en $1984^{(9)} \%$. À l'inverse de ce qui a pu être observé pour les trois autres "dragons" asiatiques, les gains engendrés par l'accès à la main d'œuvre bon marché dans le delta de la rivière des Perles ont désincité le secteur manufacturier à élever son niveau technologique. Par ailleurs, la «bulle » engendrée par la hausse vertigineuse de l'immobilier et de la bourse dans la période avant 1997 n'a pas facilité le processus d'ajustement économique.

$\mathrm{Si}$ Tung Chee-Hwa n'a pas réussi à surmonter ces difficultés, le camp démocrate n'a pas été plus à même de proposer une stratégie de développement économique. Il a également échoué à jouer le rôle d'un pouvoir d'opposition efficace et constructif. Une étude réalisée en 2001 par Lau Siu-kai montre que 63,6\% des personnes interrogées pensent que le Chef de l'exécutif n'est pas en mesure de représenter leurs opinions $(12,1 \%)$ de réponses positives. De même, $51,7 \%$ des personnes interrogées considèrent que le gouvernement de la RASHK n'est pas en mesure de représenter leurs opinions (seulement 15,2\% de réponses positives). Mais le Parti démocrate ne fait guère mieux : $46 \%$ des personnes interrogées considèrent que le parti n'est pas en mesure de représenter leurs opinions $(13,4 \%$ de réponses favorables). Les groupes d'intérêt public sont les plus à même de représenter l'opinion publique (38,6\% des personnes interrogées), avec seulement $22,1 \%$ d'avis défavorables ${ }^{(1)}$.

Le Parti démocrate et les autres groupes démocrates se plaignent régulièrement de ce que leurs propositions exposées dans des policy papers ne suscitent l'intérêt ni du gouvernement ni des médias, ni, a fortiori, celui de la population. Depuis 1997, le gouvernement de la RASHK s'est ingénié à limiter l'influence de ce qu'il considère comme un mouvement d'opposition sur le processus de décision politique. L'autocensure pratiquée par les médias, qui considèrent souvent que les propositions des démocrates ne sont pas réalistes, ne fait qu'aggraver la situation. Il faut admettre que les médias estiment que les propositions des démocrates n'auront pas beaucoup d'impact, et les délaissent pour cette raison. En tant que force d'opposition, les partis démocrates se doivent toutefois de chercher à influencer et à mobiliser la population, et plus particulièrement les groupes directement concernés par leur projet politique. Ces opérations ont des résultats mitigés, surtout en matière économique, parce que leurs idées trouvent difficilement écho auprès des milieux d'affaires. Ces derniers préfèrent pratiquer un lobbying modéré auprès du gouvernement et considèrent que la fréquentation des partis démocrates ne sert pas leurs intérêts et risque même de leur attirer les foudres de Pékin et du gouvernement hongkongais.

Sur la question des politiques sociales et des changements de valeurs, ni le gouvernement ni les partis d'opposition n'ont fait preuve de réactivité, ne cherchant pas à en comprendre les enjeux. Il s'agit d'un manque de volonté politique dans les dernières années du mandat de Tung CheeHwa et dans les années qui ont précédé la réélection de Donald Tsang. De leur côté, les partis démocrates ne se sont pas non plus révélés très efficaces dans des domaines tels que l'éducation, la couverture sociale, l'emploi des jeunes ou le développement communautaire. L'écart entre les riches et les pauvres s'est creusé sous l'effet de la mondialisation. Même les jeunes diplômés de l'enseignement supérieur sont menacés de paupérisation. Le ralentissement de la croissance économique, la réduction des effectifs de la fonction publique et du secteur public en général, la rationalisation des effectifs dans les entreprises sont autant de mesures qui viennent réduire les perspectives des jeunes cadres moyens et cadres supérieurs ${ }^{(1)}$ et remettre en question leur avenir. Ce phénomène peut favoriser le développement de la société civile comme il peut exacerber le mécontentement de la population et conduire à des mouvements de protestation. Taiwan est un cas intéressant : le processus de démocratisation entamé depuis la fin des années 1980 s'est accompagné d'un fort développement de la société civile à travers des groupes engagés dans des activités philanthropiques. Ces derniers sont capables de récolter des dons importants et de mobiliser des bénévoles qui devancent parfois les secours officiels sur les sites touchés par des catastrophes naturelles. Malgré la récente crise de confiance dont souffrent les partis politiques, le développement de la société civile n'a pas été affecté à Taiwan. Il semblerait que ni les partis politiques ni les groupes issus de la société civile n'aient réussi à exploiter ce potentiel à Hong Kong. Au contraire des années

8. Tsang Shu-ki, "Changing Structure of Hong Kong's Economy," in Gungwu Wang et John Wong (éd.), Hong Kong in China: The Challenges of Transition, Singapour, Times Academic Press, 1999, p. 108.

9. Kwong Kai-sun et al., The Impact of Relocation on Total Factor Productivity of Hong Kong Manufacturing, document réprographié, Hong Kong, Département d'économie, The Chinese University of Hong Kong, août 1997.

10. Voir Lau Siu-kai, "Socio-economic Discontent and Political Attitudes," in Siu-kai Lau et al. (eds), Indicators of Social Development: Hong Kong 2001, Hong Kong, Hong Kong Institute of Asia-Pacific Studies, The Chinese University of Hong Kong, 2003, p. 69.

11. Ohmae Kenichi, The Impact of Rising Lower-middle Class Population in Japan: What Can We Do About It? (en japonais), Tokyo, Kodansha, 2006. 
Tableau 3. Problèmes les plus fréquemment évoqués par les personnes interrogées dans le cadre des études menées par le gouvernement de Hong Kong, 1997-2003

\begin{tabular}{|c|c|c|c|c|c|c|c|c|c|c|c|}
\hline Problèmes & $\begin{array}{c}\text { Jan } \\
1997 \\
\end{array}$ & $\begin{array}{c}\text { Mar } \\
1997 \\
\end{array}$ & $\begin{array}{l}\text { Mai } \\
1997 \\
\end{array}$ & $\begin{array}{l}\text { Juil } \\
1997 \\
\end{array}$ & $\begin{array}{l}\text { Sept } \\
1997 \\
\end{array}$ & $\begin{array}{c}\text { Nov } \\
1997 \\
\end{array}$ & $\begin{array}{c}\text { Jan } \\
1998 \\
\end{array}$ & $\begin{array}{c}\text { Mar } \\
1998 \\
\end{array}$ & $\begin{array}{l}\text { Mai } \\
1998 \\
\end{array}$ & $\begin{array}{c}\text { Juil } \\
1998 \\
\end{array}$ & $\begin{array}{l}\text { Sept } \\
1998 \\
\end{array}$ \\
\hline liés au travail & $27 \%$ & $25 \%$ & $19 \%$ & $18 \%$ & $19 \%$ & $24 \%$ & $33 \%$ & $41 \%$ & $54 \%$ & $48 \%$ & $49 \%$ \\
\hline liés à l'économie & $18 \%$ & $12 \%$ & $7 \%$ & $9 \%$ & $12 \%$ & $29 \%$ & $52 \%$ & $44 \%$ & $8 \%$ & $66 \%$ & $60 \%$ \\
\hline liés à la gestion du gouvernement de la RASHK & - & - & - & - & - & - & - & - & - & - & - \\
\hline liés à la politique et à l'avenir de Hong Kong & - & - & - & - & $4 \%$ & $5 \%$ & $5 \%$ & $5 \%$ & $3 \%$ & $3 \%$ & - \\
\hline liés à l'éducation & $13 \%$ & $11 \%$ & $18 \%$ & $15 \%$ & $19 \%$ & $14 \%$ & $9 \%$ & $10 \%$ & $11 \%$ & $8 \%$ & $9 \%$ \\
\hline liés au logement & $68 \%$ & $66 \%$ & $60 \%$ & $69 \%$ & $63 \%$ & $62 \%$ & $44 \%$ & $46 \%$ & $30 \%$ & $20 \%$ & $29 \%$ \\
\hline liés à la protection s & $25 \%$ & $25 \%$ & $21 \%$ & $17 \%$ & $26 \%$ & $20 \%$ & $14 \%$ & $14 \%$ & $8 \%$ & $6 \%$ & $8 \%$ \\
\hline liés aux transports & $15 \%$ & $13 \%$ & $13 \%$ & $15 \%$ & $19 \%$ & $12 \%$ & $8 \%$ & $9 \%$ & $4 \%$ & $3 \%$ & - \\
\hline liés à la protection o & - & - & - & - & - & - & - & - & $4 \%$ & $2 \%$ & $2 \%$ \\
\hline liés à la santé & $6 \%$ & $4 \%$ & $6 \%$ & $4 \%$ & $11 \%$ & $6 \%$ & - & - & - & - & - \\
\hline liés aux nouveaux arrivants de Chine continental & ale & - & - & - & - & - & - & & $2 \%$ & - & $1 \%$ \\
\hline (Base: Nombre de personnes interrogées) & 1040 & 1045 & 1017 & 1099 & 1033 & 1033 & 1144 & 1099 & 1185 & 1272 & 1233 \\
\hline Problèmes & $\begin{array}{l}\text { Mar } \\
2000\end{array}$ & $\begin{array}{l}\text { Mai } \\
2000\end{array}$ & $\begin{array}{l}\text { Jull } \\
2000\end{array}$ & $\begin{array}{l}\text { Sept } \\
2000\end{array}$ & $\begin{array}{l}\text { Nov } \\
2000\end{array}$ & $\begin{array}{c}\text { Jan } \\
2001\end{array}$ & $\begin{array}{l}\text { Mar } \\
2001\end{array}$ & $\begin{array}{l}\text { Mai } \\
2001\end{array}$ & $\begin{array}{r}\text { Juil } \\
2001 \\
\end{array}$ & $\begin{array}{l}\text { Sept } \\
2001\end{array}$ & $\begin{array}{l}\text { Nov } \\
2001 \\
\end{array}$ \\
\hline és at & $45 \%$ & $39 \%$ & $45 \%$ & $50 \%$ & $46 \%$ & $47 \%$ & $49 \%$ & $42 \%$ & $49 \%$ & $52 \%$ & $54 \%$ \\
\hline liés à l'économie & $40 \%$ & $34 \%$ & $38 \%$ & $38 \%$ & $42 \%$ & $46 \%$ & $42 \%$ & $47 \%$ & $44 \%$ & $55 \%$ & $52 \%$ \\
\hline liés à la gestion du gouvernement de la RASHK & < - & - & - & - & - & - & - & - & - & - & $2 \%$ \\
\hline liés à la politique et à l'avenir de Hong Kong & $1 \%$ & $3 \%$ & $4 \%$ & - & - & - & - & - & - & - & $2 \%$ \\
\hline liés à l'éducation & $18 \%$ & $19 \%$ & $17 \%$ & $16 \%$ & $14 \%$ & $16 \%$ & $18 \%$ & $21 \%$ & $19 \%$ & $20 \%$ & $16 \%$ \\
\hline liés au logement & $17 \%$ & $20 \%$ & $32 \%$ & $18 \%$ & $17 \%$ & $19 \%$ & $20 \%$ & $16 \%$ & $15 \%$ & $21 \%$ & $13 \%$ \\
\hline liés à la protection sociale & $11 \%$ & $10 \%$ & $4 \%$ & $6 \%$ & $7 \%$ & $7 \%$ & $6 \%$ & $6 \%$ & $4 \%$ & $4 \%$ & $4 \%$ \\
\hline liés aux transports & - & - & - & - & - & $4 \%$ & $4 \%$ & $3 \%$ & $4 \%$ & $2 \%$ & $4 \%$ \\
\hline liés à la protection de l'environnement & $9 \%$ & $26 \%$ & $9 \%$ & $6 \%$ & $10 \%$ & $10 \%$ & $7 \%$ & $9 \%$ & $8 \%$ & $4 \%$ & $3 \%$ \\
\hline liés à la santé & 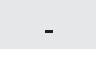 & $10 \%$ & $3 \%$ & $3 \%$ & $2 \%$ & $6 \%$ & $4 \%$ & & - & 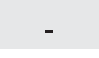 & - \\
\hline (Base: Nombre de personnes interrogées) & 1278 & 1268 & 1253 & 1154 & 1107 & 1118 & 1149 & 1157 & 1158 & 1128 & 1181 \\
\hline
\end{tabular}

1980, les syndicats étudiants se sont progressivement détournés des partis politiques démocrates.

En même temps, la préservation de sites tels que Victoria Harbour ou le Star Ferry Pier dans le quartier de Central a donné lieu à une mobilisation de la population qu'aucun des acteurs politiques de Hong Kong n'avait prévu. L'importance que revêt la préservation de ce patrimoine historique a tout simplement été sous-estimée, le gouvernement se préoccupant surtout de l'amélioration des conditions économiques: le taux de chômage est ainsi progressivement redescendu à $5 \%$ mi-2006 et le taux de croissance est passé de $1,8 \%$ en 2002 à $3,2 \%$ en 2003 , $8,6 \%$ en 2004 et $7,3 \%$ en $2005^{(12)}$. Les partis démocrates quant à eux ont été perçus comme insensibles au changement de valeurs qui s'opérait au sein de la population.

\section{Élections et réformes politiques}

Il semblerait qu'une majorité de Hongkongais ait une approche encore utilitariste envers la démocratie, qu'ils voient comme un moyen de réaliser des objectifs pratiques, concrets. Peu d'entre eux pratiquent la démocratie comme un mode de vie. La percevant comme un moyen en vue d'une fin, ils calculent avec prudence le coût de la participation politique. Si la démocratie permet de garantir les libertés, le mode de vie et le niveau de vie auxquels ils accordent tant de prix, ils estiment que les efforts individuels sont probablement plus susceptibles d'améliorer leur existence ${ }^{(13)}$ $\mathrm{Au}$ vu des grandes tendances de la mondialisation économique, il est probable que cette croyance décline et qu'ils attendent désormais un rôle accru du gouvernement.

Avec la rétrocession, la majorité des Hongkongais avaient peu ou prou accepté de troquer la démocratie contre la stabilité et la prospérité. La démocratie, perçue comme un ob-

12. Hong Kong Statistics: Understanding the Present, Planning the Future, poster publié par le Bureau des recensements et statistiques de la RAS de Hong Kong début juillet 2006.

13. Voir l'ouvrage de l'auteur, Political Participation in Hong Kong - Theoretical Issues and Historical Legacy, Hong Kong: City University of Hong Kong Press, 1999. 
Source : les enquêtes téléphoniques ont été réalisées par le Bureau des Affaires intérieures de la RASHK (ex Département des Affaires intérieures du gouvernement de Hong Kong) entre janvier 1983 et août 2003.

\begin{tabular}{|c|c|c|c|c|c|c|c|}
\hline $\begin{array}{l}\text { Nov } \\
1998\end{array}$ & $\begin{array}{c}\text { Jan } \\
1999\end{array}$ & $\begin{array}{c}\text { Mar } \\
1999\end{array}$ & $\begin{array}{l}\text { Mai } \\
1999\end{array}$ & $\begin{array}{l}\text { Juil } \\
1999\end{array}$ & $\begin{array}{l}\text { Sept } \\
1999\end{array}$ & $\begin{array}{l}\text { Nov } \\
1999\end{array}$ & $\begin{array}{c}\text { Jan } \\
2000\end{array}$ \\
\hline $50 \%$ & $46 \%$ & $49 \%$ & $47 \%$ & $45 \%$ & $38 \%$ & $47 \%$ & $51 \%$ \\
\hline $57 \%$ & $61 \%$ & $54 \%$ & $38 \%$ & $43 \%$ & $51 \%$ & $42 \%$ & $42 \%$ \\
\hline- & - & - & - & - & - & - & - \\
\hline- & - & - & - & - & $4 \%$ & $2 \%$ & $2 \%$ \\
\hline $9 \%$ & $7 \%$ & $12 \%$ & $12 \%$ & $11 \%$ & $17 \%$ & $13 \%$ & $13 \%$ \\
\hline $24 \%$ & $17 \%$ & $16 \%$ & $20 \%$ & $15 \%$ & $22 \%$ & $20 \%$ & $21 \%$ \\
\hline $9 \%$ & $12 \%$ & $10 \%$ & $6 \%$ & $7 \%$ & $10 \%$ & $7 \%$ & $7 \%$ \\
\hline- & $5 \%$ & $4 \%$ & $3 \%$ & $6 \%$ & $6 \%$ & $6 \%$ & - \\
\hline $5 \%$ & $3 \%$ & $6 \%$ & $4 \%$ & $5 \%$ & $9 \%$ & $14 \%$ & $11 \%$ \\
\hline- & - & - & - & - & - & - & - \\
\hline $1 \%$ & $2 \%$ & $14 \%$ & - & - & - & - & - \\
\hline 1296 & 1301 & 1247 & 1350 & 1219 & 1101 & 1237 & 1292 \\
\hline $\begin{array}{c}\text { Jan } \\
2002 \\
\end{array}$ & $\begin{array}{l}\text { Mar } \\
2002 \\
\end{array}$ & $\begin{array}{l}\text { Mai } \\
2002 \\
\end{array}$ & $\begin{array}{r}\text { Juil } \\
2002 \\
\end{array}$ & $\begin{array}{c}\text { Oct } \\
2002\end{array}$ & $\begin{array}{c}\text { Jan } \\
2003\end{array}$ & $\begin{array}{l}\text { Mai } \\
2003\end{array}$ & $\begin{array}{l}\text { Aou } \\
2003 \\
\end{array}$ \\
\hline $55 \%$ & $57 \%$ & $58 \%$ & $70 \%$ & $60 \%$ & $51 \%$ & $42 \%$ & $49 \%$ \\
\hline $48 \%$ & $52 \%$ & $44 \%$ & $43 \%$ & $36 \%$ & $40 \%$ & $46 \%$ & $41 \%$ \\
\hline $2 \%$ & $3 \%$ & $3 \%$ & $2 \%$ & $5 \%$ & $11 \%$ & $22 \%$ & $14 \%$ \\
\hline $2 \%$ & $2 \%$ & $3 \%$ & $3 \%$ & $7 \%$ & $6 \%$ & $4 \%$ & $8 \%$ \\
\hline $13 \%$ & $15 \%$ & $14 \%$ & $14 \%$ & $11 \%$ & $8 \%$ & $5 \%$ & $8 \%$ \\
\hline $10 \%$ & $11 \%$ & $11 \%$ & $8 \%$ & $9 \%$ & $7 \%$ & $4 \%$ & $7 \%$ \\
\hline $5 \%$ & $6 \%$ & $8 \%$ & $5 \%$ & $5 \%$ & $7 \%$ & $4 \%$ & $2 \%$ \\
\hline $2 \%$ & $3 \%$ & $2 \%$ & $3 \%$ & $5 \%$ & - & - & - \\
\hline $4 \%$ & $4 \%$ & $4 \%$ & $3 \%$ & - & - & - & - \\
\hline- & - & - & - & - & - & - & - \\
\hline 1152 & 1236 & 1237 & 1317 & 1424 & 1359 & 1447 & 1292 \\
\hline
\end{tabular}

jectif non réaliste, n'était plus une priorité, contrairement à la préservation du niveau de vie élevé dont ils bénéficient. Mais cette situation et l'état d'esprit dominant allaient se retourner brusquement. Les manifestations massives en souvenir du mouvement de Tiananmen en 1989 en témoignent. Le $1^{\text {er }}$ juillet 2003, plus d'un demi million de personnes sont descendues dans la rue pour protester contre l'article 23 et réclamer la démocratie. Les participiants aux manifestations avaient le sentiment d'écrire l'histoire et étaient fiers du calme et de l'ordre qui régnaient dans le mouvement. La crise a largement attiré l'attention des médias internationaux, ce qui en a fait une priorité pour le gouvernement chinois.

En liant la revendication démocratique et l'opposition à l'article 23 et au gouvernement Tung dans l'opinion, les manifestations du $1^{\text {er }}$ juillet 2003 ont mis du baume au cœur du mouvement démocratique. Les Hongkongais mesurèrent alors qu'ils n'avaient pas eu leur mot à dire dans la re-élection d'un Tung Chee-Hwa, que nul ne pouvait forcer à démissionner malgré son bilan déplorable. Le camp démocrate prit conscience du fait qu'il ne pouvait pas mettre des centaines milliers de manifestants dans la rue à n'importe quel moment et espéra donc utiliser les élections pour faire comprendre à Tung, à Pékin et au reste du monde que les Hongkongais ne baissaient pas les bras et appelaient la démocratie de leurs vœux.

Les élections locales de novembre 2003 furent marquées par un taux de participation record (44,1\%) (voir tableau 4). Dans la foulée des manifestations du $1^{\text {er }}$ juillet 2003, les Hongkongais se sont rendus aux urnes pour exprimer leur mécontentement et réaffirmer leur volonté de voir la démocratie instaurée. Début 2003, la plupart des observateurs s'accordaient à dire que le parti pro-gouvernemental, le DAB (Democratic Alliance for the Betterment of Hong Kong), était en position de remporter une franche victoire lors des élections locales. Le nombre de candidats présentés par le DAB reflétait aussi cette position de force (voir tableau 5). Grâce à de substantielles donations de groupes d'affaires pro-Pékin, le $\mathrm{DAB}$ avait été en mesure de développer un impressionnant réseau d'adhérents et de militants sur les dernières décennies. Les conseillers locaux et les candidats du DAB sont très présents auprès des électeurs, organisant des activités telles que banquets, pique-niques et voyages. Attentifs aux besoins de la communauté, ils offrent de nombreux services gratuits : coupe de cheveux, consultation médicale, traitements gratuits $\mathrm{du}$ SRAS, etc. Mais le soutien du DAB à l'administration Tung et sa position sur l'article 23 se transformèrent en fardeau politique. Les autres partis pro-gouvernementaux, le Liberal Party et le HKPA (Hong Kong Progressive Alliance) n'enregistrèrent que des pertes mineures (voir tableau 5).

Le Parti démocrate a pu enrayer son déclin des dernières années, gagnant 9 sièges pour passer à 95 sièges en 2003. Le parti a certainement bénéficié de la mobilisation en faveur de la démocratie exprimée lors des manifestations du $1^{\text {er }}$ juillet et du mécontentement de la population à l'égard de Tung Chee-Hwa et du DAB. Conscient de la nécessité de consolider ses forces, le Parti démocrate a présenté 120 candidats, contre 206 pour le DAB. En termes de ressources financières et humaines et en termes d'implantation locale, le nombre relativement élevé de conseillers locaux est un acquis considérable pour le Parti démocrate, qui reste le plus puissant et le plus important en nombre dans le camp démocrate. Le parti Frontier et l'ADPL (Hong Kong Association for Democracy and People's Livelihood) ont également fait de bons score aux élections locales (voir tableau 5).

On a attribué au camp des démocrates une victoire contre le DAB aux élections locales de 2003, mais en termes de répartition des forces dans les 18 conseils de district, il n'obtient la majorité que dans sept conseils, contre dix 


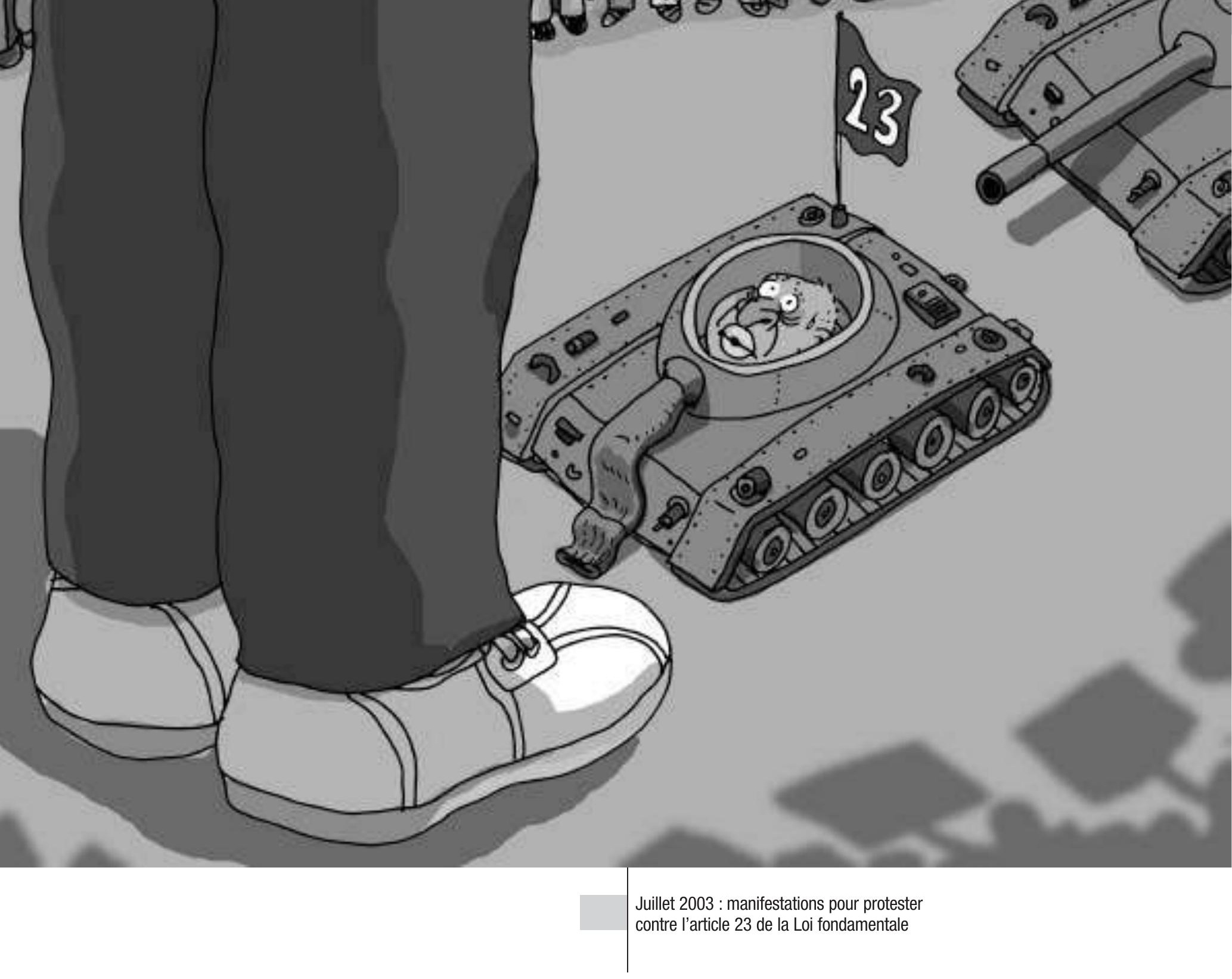

pour les partis gouvernementaux, avec une situation d'équilibre dans le district de Kowloon City. Le système des nominations a exacerbé encore cette tendance dans la mesure où il a donné la possibilité à l'administration Tung de faire pencher la balance en sa faveur dans plusieurs conseils de districts en récompensant ses fidèles. Après les nominations, seuls deux conseils de district restaient aux mains du camp démocrate et la majorité obtenue dans seize districts par le camp gouvernemental signifie que les ressources des conseils locaux échapperont largement aux démocrates. C'est donc davantage à l'aune du message politique exprimé par les votants qu'il faut comprendre la victoire en faveur de la démocratie.

Avec seulement 200 candidats pour 400 circonscriptions, le camp démocrate rencontre aussi des difficultés de recrutement politique. Parallèlement, les actions menées en termes de services de proximité par le DAB auprès de sa base électorale devraient à nouveau se traduire par de bons résultats aux élections locales de 2007.

Le mouvement démocrate n'a que partiellement réussi à réitérer son succès passé lors des élections législatives de sep- tembre 2004. Le taux de participation a atteint un nouveau record de $55,6 \%$, ce qui représente une augmentation de $12 \%$ par rapport à 2000. Ce sont 1,78 millions de Hongkongais qui se sont rendus aux urnes (voir tableau 6). Si l'on considère le nombre de sièges obtenus, le camp démocrate n'a pas fait un très bon score : 18 élus dans les circonscriptions géographiques, soit deux de plus qu'en 2000, et 7 dans le système des circonscriptions fonctionnelles (corporations professionnelles et syndicats), soit deux de plus qu'en 2000 (voir tableau 7). Des résultats qui auraient pu être meilleurs ${ }^{(14)}$.

Le camp démocrate a probablement souffert de scandales, de l'hostilité des médias et des campagnes de dénigrement. Il est passé de $58,2 \%$ des suffrages en 2000 à $60,5 \%$ en 2004, un score malgré tout légèrement inférieur à celui de $1998(63,2 \%)^{(15)}$. En regard du taux de participation record et de l'impressionnante mobilisation de la population lors

14. Voir l'article de l'auteur "Hong Kong's Democrats Stumble," art. cit., pp. 138-152.

15. Ces chiffres varient légèrement suivant le nombre de candidats considérés comme faisant partie du camp démocrate. Les spécialistes s'opposent en effet sur l'appartenance politique d'un ou deux candidats controversés ou marginaux. 
Tableau 4. Nombre d'électeurs et taux de participation aux élections locales, 1982-2003

\begin{tabular}{|c|c|c|c|c|c|}
\hline & $\begin{array}{l}\text { Nombre de com- } \\
\text { missions de district } \\
\text { / circonscriptions* }\end{array}$ & $\begin{array}{c}\text { Nombre de } \\
\text { sièges à pourvoir }\end{array}$ & $\begin{array}{l}\text { Nombre } \\
\text { d'électeurs } \\
\text { inscrits }\end{array}$ & $\begin{array}{c}\text { Nombre } \\
\text { d'électeurs } \\
\text { ayant voté ** }\end{array}$ & $\begin{array}{c}\text { Taux de } \\
\text { participation } \\
\text { électorale** }^{*}\end{array}$ \\
\hline 2003 & 400 & 400 & 2973612 & 1065363 & $44,1 \%$ \\
\hline 1999 & 390 & 390 & 2832524 & 816503 & $35,8 \%$ \\
\hline 1994 & 346 & 346 & 2450372 & 693215 & $33,1 \%$ \\
\hline 1991 & 209 & 272 & 1840413 & 423923 & $32,5 \%$ \\
\hline 1988 & 157 & 264 & 1610998 & 424201 & $30,3 \%$ \\
\hline 1985 & 145 & 237 & 1421391 & 476558 & $37,5 \%$ \\
\hline 1982 & 122 & 132 & 899559 & 342764 & $38,1 \%$ \\
\hline Notes: & $\begin{array}{l}\text { *Avant 1997, les conseils de distric } \\
\text { élisaient deux membres chacunes } \\
\text { ** Certains candidats étant élus san } \\
\text { les taux de participation électoral }\end{array}$ & $\begin{array}{l}\text { appelés commissions de dis } \\
\text { tion, un certain nombre d'él }\end{array}$ & rs des électio & avant, certain & scriptions \\
\hline
\end{tabular}

Source: Voir l'article de l'auteur "The 2003 District Council Elections in Hong Kong," Asian Survey, vol. 44, $n^{\circ}$ 5, septembre/octobre 2004, pp. 734-754.

Tableau 5. Résultats des principaux partis politiques aux élections locales, 1994-2003

\begin{tabular}{|c|c|c|c|c|c|}
\hline & $\begin{array}{c}\text { Nombre de } \\
\text { candidats } \\
\text { en } 2003\end{array}$ & $\begin{array}{l}\text { Nombre } \\
\text { de sièges } \\
\text { obtenus } \\
\text { en } 2003\end{array}$ & $\begin{array}{c}\% \text { de sièges } \\
\text { obtenus en } \\
2003\end{array}$ & $\begin{array}{c}\text { Nombre de } \\
\text { sièges } \\
\text { obtenus en } \\
1999\end{array}$ & $\begin{array}{c}\text { Nombre de } \\
\text { sièges } \\
\text { obtenus en } \\
1994\end{array}$ \\
\hline Democratic Party & 120 & 95 & 79,2 & 86 & 75 \\
\hline DAB & 206 & 62 & 30,1 & 83 & 37 \\
\hline ADPL & 37 & 25 & 67,6 & 19 & 29 \\
\hline Liberal Party & 25 & 13 & 52,0 & 15 & 18 \\
\hline The Frontier & 13 & 6 & 46,1 & 4 & 0 \\
\hline $\begin{array}{l}\text { Hong Kong Progressive } \\
\text { Alliance }\end{array}$ & 38 & 20 & 52,6 & 21 & 2 \\
\hline
\end{tabular}

des manifestations du $1^{\text {er }}$ juillet 2003 et du $1^{\text {er }}$ juillet 2004, ce résultat est légèrement décevant. Les partis pro-gouvernementaux ont réussi à maintenir une majorité au sein du Legco tandis que les partis démocrates sont restés dans l'opposition : une conséquence directe du système électoral qui empêche les premiers d'avoir une majorité au Legco en dépit de l'obtention d'une majorité absolue aux élections directes. Les élections législatives de 2004 n'ont pas non plus occasionné de débats sur la valeur de ces élections et les importantes questions politiques qui en découlent.

C'est dans ce contexte que Donald Tsang a proposé son projet de réforme politique qui prévoit l'ajout de cinq sièges élus au suffrage universel et cinq sièges de circonscriptions fonctionnelles au Legco en 2008. Ces derniers seraient choisis par les conseillers locaux, le camp démocrate pourrait donc espérer récupérer deux ou trois sièges parmi les sièges sup- 
Tableau 6. Nombre d'électeurs et taux de participation électorale aux élections législatives, 1991-2004

\begin{tabular}{|c|c|c|c|c|c|c|c|c|c|c|c|c|c|c|c|}
\hline & \multicolumn{5}{|c|}{ Circonscriptions géographiques } & \multicolumn{5}{|c|}{ Circonscriptions fonctionnelles } & \multicolumn{5}{|c|}{ Elus par le Comité électoral } \\
\hline & $\begin{array}{c}\text { Nombre de } \\
\text { candidatures } \\
\text { valides }\end{array}$ & $\begin{array}{l}\text { Nombre } \\
\text { de } \\
\text { sièges }\end{array}$ & $\begin{array}{c}\text { Nombre } \\
\text { d'électeurs } \\
\text { inscrits }\end{array}$ & $\begin{array}{c}\text { Nombre } \\
\text { d'électeurs } \\
\text { ayant voté }\end{array}$ & 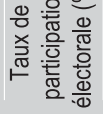 & $\begin{array}{c}\text { Nombre de } \\
\text { candidatures } \\
\text { valides }\end{array}$ & $\begin{array}{l}\text { Nombre } \\
\text { de } \\
\text { sièges }\end{array}$ & $\begin{array}{l}\text { Nombre } \\
\text { d'électeurs } \\
\text { inscrits }\end{array}$ & $\begin{array}{c}\text { Nombre } \\
\text { d'électeurs } \\
\text { ayant voté }\end{array}$ & 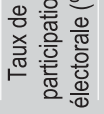 & $\begin{array}{c}\text { Nombre de } \\
\text { candidatures } \\
\text { valides }\end{array}$ & $\begin{array}{l}\text { Nombre } \\
\text { de } \\
\text { sièges }\end{array}$ & $\begin{array}{l}\text { Nombre } \\
\text { d'électeurs } \\
\text { inscrits }\end{array}$ & $\begin{array}{l}\text { Nombre } \\
\text { d'électeurs } \\
\text { ayant voté ' }\end{array}$ & 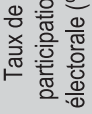 \\
\hline 2004 & 88 & 30 & 3207227 & 1784406 & 55,64 & 71 & 30 & 192374 & 134852 & 70,10 & - & - & - & - & - \\
\hline 2000 & 36 & 24 & 378 & & 43,57 & 57 & 30 & 163026 & 92112 & 56,50 & 10 & 6 & 783 & 748 & 95,53 \\
\hline 1998 & 81 & 20 & 2795371 & 1489705 & 53,29 & 60 & 30 & 122537 & 77813 & 63,50 & 25 & 10 & 80 & 790 & 98,75 \\
\hline 1995 & 50 & 20 & $2572135^{a}$ & 920567 & 35,79 & 70 & 30 & 1139835 & 460690 & 40,42 & 18 & 10 & 283 & 282 & 99,65 \\
\hline 1991 & 54 & 18 & 1916925 & 750476 & $39,15^{b}$ & 40 & 21 & $48756^{c}$ & 22919 & 47,01 & - & 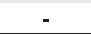 & - & 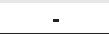 & \\
\hline
\end{tabular}

Notes : a Nombre approximatif d'électeurs inscrits calculé à partir du nombre d'électeurs ayant voté et du taux de participation électorale.

b Chiffre officiel du gouvernement. Les spécialistes et les médias considèrent que ce chiffre est sous-évalué et que le véritable taux de participation pourrait atteindre $47.5 \%$ à 51.8\%. Voir, par exemple, Louie Kin-sheun, et al., "Who Voted in the 1991 Elections? A Socio-Demographic Profile of the Hong Kong Electorate", in Lau Siu-kai and Louie Kin-sheun (eds.), Hong Kong Tried Democracy: The 1991 Elections in Hong Kong, Hong Kong: The Chinese University of Hong Kong, 1993, pp. 1-39; and Rowena Kwok, Joan Leung and lan Scott (eds.), Votes Without Power: The Hong Kong Legislative Council Elections 1991, Hong Kong: Hong Kong University Press, 1992, p. 8.

c Comprend le nombre d'électeurs inscrits pour les circonscriptions en lice. Le nombre total d'électeurs inscrits pour les circonscriptions fonctionnelles était de 69825 . 12 des 21 sièges n'étaient pas concernés par l'élection.

Source : Commission des Affaires électorales du gouvernement de la RASHK, Report on the 2004 Legislative Council Elections, 11 décembre 2004, (http://www.eac.gov.hk/en/legco/2004_report.htm, 28 février 2007), Commisssion des Affaires électorales du gouvernement de la RASHK, Report on the 2000 Legislative Council Elections, 2000, (http://www.eac.gov.hklen/legco/2000_report.htm, 28 février 2007); Commission des Affaires électorales du gouvernement de la RASHK, Report on the 1998 Legislative Council Elections, 18 septembre 1998, (http:///Www.info.gov.hklinfo/98eac-e.htm, 28 février 2007); Hsin-chi Kuan et al. (eds), The 1995 Legislative Council Elections in Hong Kong, Hong Kong: The Chinese University of Hong Kong, 1996, pp. 2 and 21; Rowena Kwok et al. (eds), The Chinese University of Hong Kong, 1993, pp. 32 and 157.

plémentaires de circonscriptions fonctionnelles ${ }^{(16)}$. D'après la Loi fondamentale de Hong Kong, une réforme du système est en effet prévue pour les élections après 2007, d'où le projet proposé par l'Administration Tsang. Refusant le projet de réforme intermédiaire du gouvernement, le camp démocrate réclame un calendrier et une feuille de route pour l'instauration du suffrage universel pour les élections du Legco et du Chef de l'exécutif.

Cependant, tout projet de réforme doit être avalisé par les deux tiers du Legco, ce qui permet au camp démocrate d'exercer un droit de veto. Encouragé par une grande manifestation (250000 personnes selon les organisateurs, 63000 selon la police) organisée le 4 décembre $2004^{(17)}$ contre le projet de réforme politique, le camp démocrate réussit à maintenir une cohésion dans ses rangs et à contrer les tentatives du camp de Tsang pour gagner l'adhésion de six d'entre eux afin d'atteindre les deux tiers requis pour l'approbation du projet.

Si le rejet du projet de réforme par vingt-quatre députés est perçu comme un échec cuisant pour l'administration Tsang, il ne s'agit pas vraiment d'une victoire pour le camp démocrate car l'opinion publique était favorable au projet. Cet affrontement a conduit les autorités chinoises à revoir leur position de relative tolérance à l'égard du camp démocrate depuis les élections législatives de septembre 2004. Aucune autre réforme politique n'étant envisageable dans un futur prévisible, les leaders démocrates de la nouvelle génération voient donc leurs perspectives considérablement restreintes.
Sans possibilité réelle de faire une nouvelle percée, le camp démocrate s'essouffle.

\section{Défis à venir}

Au cours des campagnes électorales qui se sont succédé ces dix dernières années, le mouvement pour la démocratie a fait ses preuves, s'assurant généralement un score de $60 \%$ aux élections législatives directes. Cela s'explique par la volonté d'une majorité d'électeurs hongkongais de maintenir un équilibre des pouvoirs en élisant une majorité de démocrates pour ne pas laisser libre-cours au gouvernement. Mais, dans le cadre du système électoral actuel et en l'absence de réformes démocratiques, il semble difficilement envisageable que le camp démocrate obtienne une majorité de sièges au Legco. Par conséquent, il ne constitue pas une alternative crédible aux yeux de la population. Les efforts engagés en ce sens ne se sont pas révélés suffisants : le camp démocrate n'a par exemple pas été capable de désigner un cabinet fantôme. La candidature d'Alan Leong au poste de Chief Executive en 2007 constitue une tentative de proposer une véritable plateforme politique, mais sur laquelle les débats sont restés limités.

16. Concernant le projet de réforme politique, voir le site internet du Bureau de la réforme constitutionnelle du gouvernent de le RASHK (http://www.cab.gov.hk/cd/ eng/past/index.htm, 28 Février 2007). Voir aussi tous les grands quotidiens du 22 décembre 2005.

17. South China Morning Post, 5 et 6 décembre 2005 
Table 7. Résultat des principaux partis politiques aux élections législatives en termes de sièges obtenus, 1991-2004

\begin{tabular}{|c|c|c|c|c|c|c|c|c|c|c|c|c|c|c|}
\hline & \multicolumn{2}{|c|}{1991} & \multicolumn{4}{|c|}{1995} & \multicolumn{3}{|c|}{1998} & \multicolumn{3}{|c|}{2000} & \multicolumn{2}{|c|}{2004} \\
\hline & CG & CF & CG & $\begin{array}{c}\text { New } \\
\text { CF }\end{array}$ & $\begin{array}{l}\text { Old } \\
\text { CF }\end{array}$ & EC & CG & CF & EC & CG & CF & EC & CG & CF \\
\hline Democratic Party & $14^{*}$ & 2 & 12 & 2 & 3 & 2 & 8 & 4 & - & 9 & 3 & - & 7 & 2 \\
\hline DAB/HKFTU & - & 1 & 2 & 2 & 2 & 2 & 5 & 3 & 3 & 6 & 4 & 1 & 9 & 3 \\
\hline ADPL & 1 & - & 2 & - & 1 & 1 & - & - & - & 1 & - & - & 1 & - \\
\hline Liberal Party & - & - & 1 & 3 & 6 & - & - & 8 & - & - & 8 & - & 2 & 8 \\
\hline The Frontier & - & - & - & - & - & - & 2 & - & - & 2 & - & - & 1 & - \\
\hline $\begin{array}{l}\text { Hong Kong Progressive } \\
\text { Alliance }\end{array}$ & - & - & - & - & - & 2 & - & 2 & 2 & 1 & 1 & 2 & - & - \\
\hline Article 45 Concern Group & - & - & - & - & - & - & - & - & - & - & - & - & 3 & 1 \\
\hline
\end{tabular}

Notes: $\quad$ 1. $\quad C G=$ Circonscriptions géographiques

$\mathrm{CF}=$ Circonscriptions fonctionnelles

$E C=$ Elus par Comité électoral

2. Le DAB est né en 1992; Liberal Party, 1993; Democratic Party, 1994; Hong Kong Progressive Alliance, 1994;

The Frontier, 1996; et Article 45 Concern Group, 2003. Le DAB et la Hong Kong Progressive Alliance ont fusionn en 2005 pour donner naissance à la Democratic Alliance for the Betterment and Progress of Hong Kong.

3. * United Democrats of Hong Kong obtient 12 sièges aux élections, tandis que le Meeting Point obtient 2 sièges. Les deux partis ont fusionné en 1994 pour former l'actuel Democratic Party.

Sources: $\quad$ Joseph Y.S. Cheng, "Hong Kong's Democrats Stumble," Journal of Democracy, vol. 16, n 1, 2005, pp. 138-152; Hsin-chi Kuan et al. (eds), The 1995 Legislative Council Elections in Hong Kong. Hong Kong: The Chinese University of Hong Kong, 1996, p. 3; Siu-kai Lau and Kin-sheun Louie (eds), Hong Kong Tried Democracy: The 1991 Elections in Hong Kong, Hong Kong: The Chinese University of Hong Kong, 1993, p. 158; Rowena Kwok et al. (eds), Votes Without Power: The Hong Kong Legislative Council Elections 1991, Hong Kong: Hong Kong University Press, 1992, pp. 5 and 212-219; Bases de données du Public Governance Programme, Lingnan University, (http://www.In.edu.hk/pgp/level2/pgp-data-f.htm, 28 févrie 2007).

La plupart des Hongkongais acceptent désormais l'idée que le gouvernement chinois est décisionnaire sur la question des réformes politiques. Ils ne souhaitent pas entrer en conflit avec les autorités chinoises et comprennent qu'en l'absence de démocratie en Chine, il ne faut pas attendre de véritable démocratie à Hong Kong. Depuis un an ou deux, les nouveaux dirigeants chinois ont intensifié leur contrôle sur les médias, les services d'information par Internet, les dissidents, ou encore les organisations non gouvernementales. Les réformes politiques ne semblent pas à l'ordre du jour en Chine. Sur le long terme, l'optimisme est plus répandu, sur la foi de l'idée que les réformes économiques ouvriront la voie aux réformes politiques.

Par ailleurs, les autorités chinoises ont refusé d'engager officiellement le dialogue avec les partis démocrates. Il s'agit en partie d'une sanction en raison du rôle qu'ont joué ces groupes lors des évènements de Tiananmen et des tentatives de ces derniers pour obtenir une révision de la version offi- cielle de ces évènements. Mais cette position s'inscrit aussi plus généralement dans la campagne de dénigrement des groupes démocrates par les autorités chinoises. Elles entretiennent malgré tout des contacts informels avec les activistes, espérant ainsi obtenir des informations et gagner à leur cause ceux qui peuvent l'être, selon le principe du front uni. De leur côté, les groupes démocrates ne sont pas non plus ouverts au dialogue. La cohésion et la coordination nécessaires à la mise au point d'une stratégie commune de négociation avec les autorités chinoises leur fait défaut. Leur impuissance à prévenir les fuites d'informations confidentielles dans les médias est célèbre. Il en résulte une certaine tendance à camper sur ses positions et à percevoir toute forme de flexibilité ou de concession comme une trahison. Leurs réactions au projet de réforme politique proposé par Donald Tsang illustrent parfaitement ce phénomène.

Les autorités chinoises sont en position de force et n'ont nulle intention de négocier avec les groupes démocrates, 


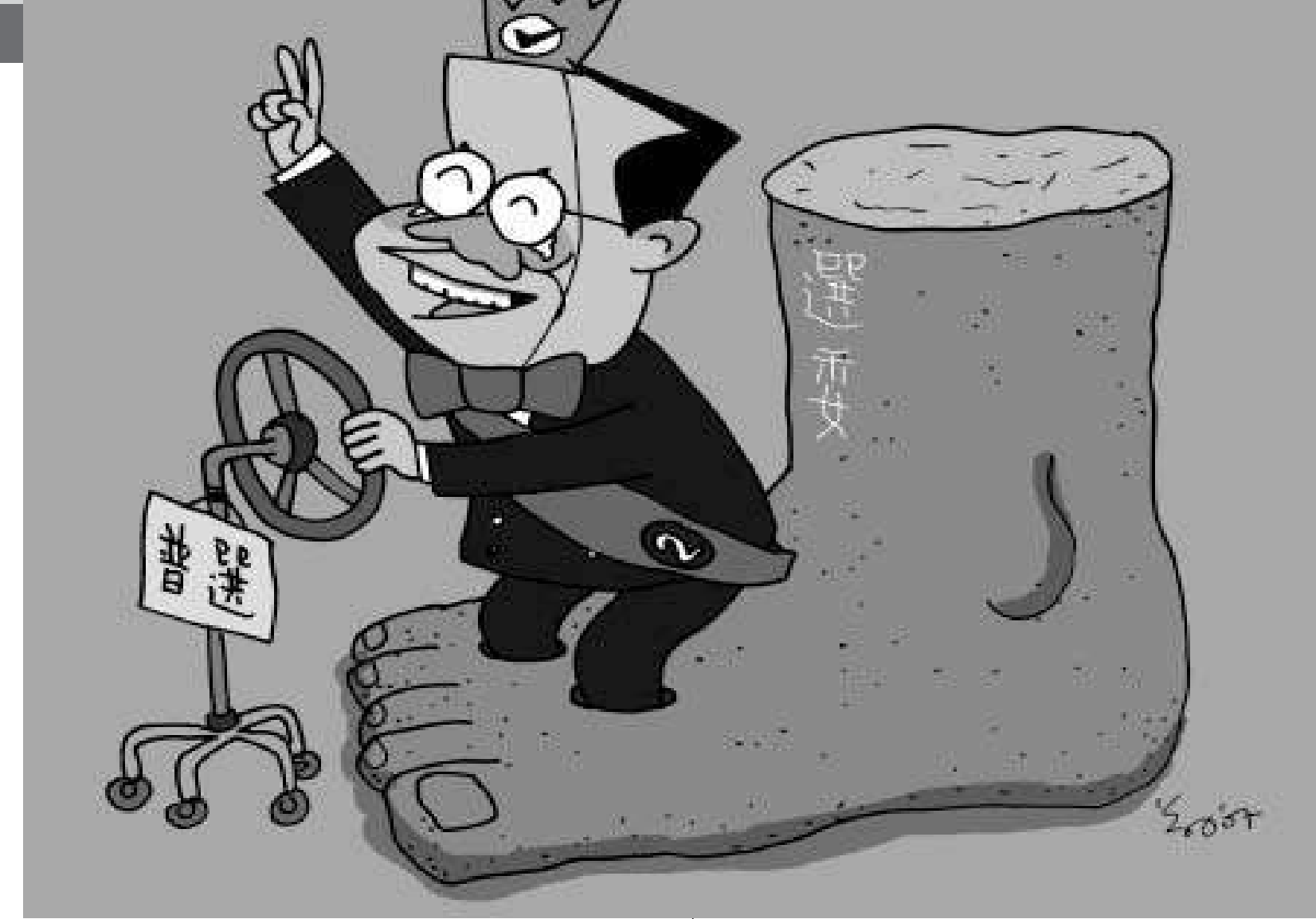

En mars 2007, Donald Tsang a été réélu à la tête du territoire sans avoir proposé d'échéancier clair en matière de suffrage universel

qu'elles traitent dans le cadre du front uni, de même que l'ensemble des groupes politiques de Hong Kong. Leur objectif est de faire accepter les bases de leur politique à Hong Kong aux groupes démocrates. Cette acceptation serait sans doute récompensée par un traitement semblable à celui des " partis démocratiques » en Chine. Mais ce pacte signifierait un suicide politique, même pour les partis pro-Pékin. L'ADPL, qui a choisi de rester dans le parlement provisoire non-élu instauré en 1997 au lieu de le boycotter comme les autres partis démocrates, a perdu ses 4 sièges au Legco aux élections législatives de 1998 (voir tableau 7). En s'alignant sur la ligne de Pékin, le HKPA (Hong Kong Progressive Alliance) a aussi causé sa perte : il a perdu ses quatre sièges au Legco en 2004 et a fusionné avec le DAB l'année suivante (voir tableau 7 ).

Suivant la logique du front uni, les partis les plus virulents à l'égard de la politique de Pékin feront, à titre d'exemple, l'objet de sanctions particulières. C'est le cas du Civic Party, issu du "Groupe pour l'article 45 " et officiellement lancé en mars 2006. Pour avoir rejeté le projet de réforme de Donald Tsang, il fait l'objet d'une campagne de sape qui a pris la forme de pressions secrètement exercées sur les milieux d'affaires pour les dissuader de faire des donations au parti nouvellement formé. Les médias pro-Pékin se sont acharnés sur le Civic Party, relâchant même pour l'occasion la pression sur le Parti démocrate.
La tactique de front uni de Pékin a échoué à diviser de façon significative le camp démocrate, même si la cohésion au sein du mouvement en a été sévèrement menacée. La pression de l'opinion publique, combinée avec une certaine pression morale, qui avait permis le maintien de la cohésion au sein du mouvement avant la rétrocession, s'est rapidement relâchée après juillet 1997. Dans un tel contexte, les divergences d'orientations politiques exacerbées se firent rapidement jour. Ces divergences portaient à l'origine sur trois points : 1) les relations avec les autorités chinoises et le gouvernement de la RASHK, 2) la nécessité de traiter uniformément les intérêts de classes ou de traiter de façon distincte les intérêts des travailleurs et de la base, 3 ) la nécessité d'agir dans le cadre du Legco ou de s'appuyer sur des mouvements de masse en dehors de tout cadre politique.

À l'heure actuelle, le parti Frontier refuse toujours de prendre part aux élections des circonscriptions fonctionnelles du Legco. Lors de l'élection de 2007, la campagne d'Alan Leong a principalement été soutenue par le Civic Party et le Parti démocrate. Le parti Frontier, la Hong Kong Confederation of Trade Unions (HKCTU), le Neighbourhood and Worker's Service Centre ont refusé de prendre part à cette élection "en comité restreint». Un parti nouvellement formé, la League of Social Democrats, s'est même activement opposé à la campagne d'Alan Leong. 
Tableau 8. Popularité des conseillers législatifs pro-démocratie, 1998-2007

Les chiffres indiquent le nombre de conseillers législatifs pro-démocratie parmi les « dix plus populaires », les « cinq plus populaires », les « trois plus populaires » et « le plus populaire».

\begin{tabular}{|c|c|c|c|c|}
\hline Mois & 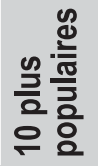 & 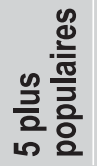 & 을 & $\begin{array}{l}\text { 을 } \\
\text { 을 } \\
\text { 을응 } \\
\text { 을 }\end{array}$ \\
\hline
\end{tabular}

\begin{tabular}{lllll}
\hline Janvier 2007 & 6 & 2 & 1 & 0 \\
\hline Octobre 2006 & 6 & 2 & 1 & 0 \\
\hline Juillet 2006 & 6 & 2 & 1 & 0 \\
\hline Avril 2006 & 6 & 2 & 1 & 0 \\
\hline Janvier 2006 & 6 & 1 & 1 & 0 \\
\hline Novembre 2005 & 6 & 2 & 1 & 0 \\
\hline August 2005 & 6 & 2 & 0 & 0 \\
\hline Mai 2005 & 6 & 3 & 1 & 0 \\
\hline Février 2005 & 6 & 2 & 1 & 0 \\
\hline Novembre 2004 & 6 & 1 & 1 & 1 \\
\hline August 2004 & 7 & 2 & 1 & 1 \\
\hline Mai 2004 & 6 & 2 & 1 & 1 \\
\hline Février 2004 & 5 & 2 & 1 & 1 \\
\hline Novembre 2003 & 6 & 2 & 1 & 1 \\
\hline August 2003 & 5 & 2 & 1 & 1 \\
\hline Mai 2003 & 5 & 2 & 1 & 0 \\
\hline Février 2003 & 4 & 2 & 0 & 0 \\
\hline Novembre 2002 & 5 & 3 & 2 & 1 \\
\hline August 2002 & 5 & 3 & 1 & 1 \\
\hline Mai 2002 & 5 & 2 & 1 & 0 \\
\hline Février 2002 & 5 & 2 & 2 & 1 \\
\hline Novembre 2001 & 5 & 2 & 2 & 1 \\
\hline August 2001 & 6 & 3 & 2 & 1 \\
\hline Mai 2001 & 5 & 2 & 2 & 0 \\
\hline Février 2001 & 5 & 3 & 2 & 0 \\
\hline Novembre 2000 & 5 & 2 & 2 & 0 \\
\hline August 2000 & 6 & 4 & 3 & 1 \\
\hline Mai 2000 & 6 & 4 & 3 & 1 \\
\hline Mars 2000 & 6 & 4 & 3 & 1 \\
\hline Janvier 2000 & 4 & 2 & 1 & 0 \\
\hline Novembre 1999 & 5 & 2 & 2 & 1 \\
\hline Septembre 1999 & 7 & 4 & 2 & 1 \\
\hline Juillet 1999 & 6 & 3 & 2 & 1 \\
\hline Mai 1999 & 6 & 3 & 3 & 1 \\
Mars 1999 & 6 & 5 & 3 & 1 \\
\hline Janvier 1999 & 6 & 5 & 3 & 1 \\
\hline Novembre 1998 & 6 & 5 & 3 & 1 \\
\hline Septembre 1998 & 6 & 5 & 3 & 1 \\
\hline Juillet 1998 & 6 & 5 & 3 & 1 \\
\hline Sore st & & \\
\hline
\end{tabular}

Source: Site internet du Public Opinion Programme de l'Université de Hong Kong, POP Polls: Rating of Top Ten Legislative Councillors. (http://hkupop.hku.hk/english/popexpress//crating/topten2.html, 28 février 2007).
Ces divergences d'orientation politique sont encore plus marquées au sein même du Parti démocrate. Les attentes des « jeunes Turcs » les ont conduit à défier la direction du parti en décembre $1998^{(18)}$. Les " jeunes Turcs " et les factions marginales du parti s'opposaient aux efforts engagés pour améliorer les relations avec Pékin, qui consistaient notamment à présenter des candidats à l'ANP (Assemblée nationale populaire) chinoise. Ils cessèrent de se montrer conciliants envers le gouvernement de la RASHK et appelèrent publiquement à la démission de Tung Chee $\mathrm{Hwa}$. Ils mirent enfin en garde les dirigeants du Parti démocrate contre l'opportunisme consistant à vouloir représenter les intérêts de l'ensemble des classes. En retour, ils furent accusés de vouloir transformer le parti démocrate en parti travailliste et d'adopter une approche populiste. Par-dessus tout, les " jeunes Turcs » et les factions marginales voulaient un retour à la base pour mobiliser les masses plutôt que de futiles discussions parlementaires.

Les divergences au sein du Parti démocrate sont à l'origine de plusieurs vagues de démissions de la part des membres les plus radicaux. Ces turbulences ont nui à l'image du parti. Les autres partis démocrates ont rencontré des difficultés avec les mouvements associatifs nées dans les années 1960 et 1970. Ces mouvements populaires ont soutenu les partis démocrates, qui pouvaient se faire le relais de leurs idées au sein du Legco ou agir comme un levier efficace auprès de l'administration Tung, mais les partis ont souvent retardé la résolution des problèmes et échoué à trouver un compromis en raison de leur tendance à l'autopromotion.

Craignant d'être instrumentalisées et davantage préoccupées par la résolution de problèmes concrets que par les intrigues politiques, de nombreuses organisations populaires ont préféré se passer du soutien des partis politiques. Les groupes pro-Pékin, ajustant visiblement leur stratégie de front uni, les ont également courtisées.

$\mathrm{Au}$ cours de la dernière décennie, une nouvelle génération d'activistes politiques a assumé des responsabilités dans de nombreuses associations politiques locales. Cette nouvelle génération s'intéresse moins à l'instauration de la démocratie à Hong Kong qu'aux problèmes environnementaux, au développement communautaire, aux droits des homosexuels, aux abus au travail, à l'aide aux groupes les plus défavorisés etc. Ils adoptent des positions plus radicales. Pour eux, les partis du camp démocrate font partie de l'ordre établi. Ils

18. Ivan Chi-keung Choy, "A Coup in the Frosty Month - Counter-attack by the Non-mains tream Factions of the Parti démocrate (en chinois)," Ming Pao (quotidien en langue chinoise de Hong Kong), 16 décembre 1998 
voient d'un mauvais oeil la priorité accordée par ces derniers aux travaux parlementaires et accusent de nombreux dirigeants démocrates d'être uniquement préoccupés par leur carrière. Leur combat pour les droits des homosexuels et des prostitués pose parfois des problèmes pour la participation de groupes religieux conservateurs au mouvement.

Les divergences entre les partis du camp démocrate, au sein même du Parti démocrate et entre les partis pro-démocrates et les organisations populaires sont parfois insignifiantes ou créées par des inimitiés personnelles. Il n'en demeure pas moins qu'elles ont été sources de déception pour les militants, ont attiré l'attention des médias et nui à l'image de toutes les parties concernées.

C'est dans ce contexte que, début 2002, un certain nombre de nouveaux groupes tels que Power for Democracy, Hong Kong Democratic Development Network et Civil Human Rights Front sont nés. Donnant la priorité à l'instauration de la démocratie et à la cause des droits de l'homme, ils souhaitaient offrir une alternative aux partis traditionnels en termes de participation politique. L'émergence et le développement de ces nouveaux groupes reflétaient le désenchantement ressenti à l'égard des partis politiques et des leaders du camp démocrate. Il est significatif que ces nouveaux groupes soient dirigés par des activistes religieux ou des intellectuels dont on considère qu'ils n'ont a priori pas d'ambition politique. Ces nouveaux groupes ont tenté de mobiliser diverses organisations favorables à la démocratie et aux droits de l'homme à l'occasion du mouvement contre l'article 23. Cette initiative intervenait dans le contexte du déclin général de l'attractivité des partis démocrates dû aux problèmes évoqués plus haut. La crise de confiance dont souffrent les partis nuit au processus d'instauration de la démocratie dont ils devraient être les principaux acteurs. De plus, les groupes émergents bénéficient de ressources très limitées et les partis rechignent à suivre leur voie. La nouvelle génération d'activistes politiques a pris les rênes du Civil Human Rights Front, rendant désormais problématiques ses relations avec les partis politiques. Le groupe Power for Democracy intervient toujours efficacement dans la coordination des campagnes électorales du camp démocrate, comme lors des élections locales de 2003 et 2007 et des élections législatives de 2004.

À l'heure actuelle, et dans un futur proche, le mouvement pour la démocratie doit surmonter trois difficultés. Contrairement au $\mathrm{DAB}$ ou au Liberal Party, les partis démocrates n'ont pas su recruter suffisamment d'adhérents. Les cadres et hommes d'affaires, qui entretiennent des relations de plus en plus étroites avec la Chine continentale, hésitent à s'associer à eux. Plus grave : la plupart des leaders prendront bien- tôt leur retraite (voir tableau 8) et la nouvelle génération ne semble pas très prometteuse car peu d'efforts ont été consentis pour assurer la relève.

D'une façon générale, les partis du camp démocrate, Civic Party compris, ont des problèmes de financement. Le modèle taiwanais, dans lequel les entreprises font des dons à tous les partis politiques proportionnellement à leur importance, fait pâlir d'envie tous les démocrates de Hong Kong. Les conseillers de district affiliés aux partis démocrates doivent normalement contribuer au fonctionnement de leurs quartiers généraux respectifs à hauteur d'un dixième de leur salaire, alors que les conseillers de district affilié au DAB reçoivent une allocation mensuelle de 4000 US $\$$ pour leur travail d'élus. Cette disparité reflète les difficultés financières des partis d'opposition et explique la qualité des services proposés par le DAB à ses électeurs.

Les partis politiques de Hong Kong sont des " partis de cadres ». Ils sont donc très dépendants des médias pour tout ce qui touche à leur image et à leur attractivité auprès du public. L'autocensure pratiquée par les médias locaux leur est donc particulièrement dommageable. Les médias sont en grande partie aux mains de grands groupes d'affaires qui ont, pour la quasi-totalité d'entre eux, des activités en Chine continentale, d'où la propension des médias à ménager Pékin. Pire encore, certains grands patrons utilisent les médias qu'ils détiennent pour faire prospérer leurs affaires avec Pékin. Les autorités chinoises ont pour leur part parfaitement compris comment utiliser les médias pour contenir le mouvement pour la démocratie. C'est ainsi qu'un grand nombre de patrons se sont vu proposer de devenir membres du Comité National de la Conférence Consultative Politique du Peuple Chinois.

Si le problème de l'autocensure ne se pose pas pour les médias internationaux, Hong Kong, qui, depuis 1997, n'est plus qu'une " région administrative spéciale » ne peut pas espérer attirer leur attention autant que par le passé. Les gouvernements occidentaux désireux d'agir pour la démocratie depuis Hong Kong sont de moins en moins nombreux car ils entretiennent des liens de plus en plus forts avec la Chine. Les groupes démocrates de Hong Kong sont extrêmement prudents, notamment dans leurs contacts avec Taiwan et les Etats-Unis.

\section{Conclusion}

Depuis la rétrocession, les attentes des Hongkongais en matière de démocratie ont été revues à la baisse. Par rapport à l'espoir qu'avaient fait naitre les dernières années de l'admi- 
nistration coloniale britannique, le mouvement pour la démocratie est désormais limité par un environnement nettement plus hostile. Un renouveau est intervenu avec l'échec du gouvernement de Tung Chee Hwa et la crise de l'article 23. Mais cette percée a révélé les restrictions imposées par le système électoral et la promptitude des autorités chinoises à intervenir pour défendre leurs intérêts fondamentaux et leur position sur Hong Kong. Le mouvement pour la démocratie est donc face à un dilemme : chercher la reconnaissance des autorités chinoises équivaut à un suicide politique, mais une position de défi à leur égard risque de provoquer un interventionnisme accru. Néanmoins, leur réponse est claire : assurer la pérennité du mouvement dans la perspective de l'instauration de la démocratie en Chine.

La survie du mouvement pour la démocratie est heureusement garantie par deux éléments : tous les acteurs politiques concernés sont conscients de la nécessité de maintenir l'État de droit et la liberté d'information pour permettre à Hong Kong de conserver son statut de centre financier et plateforme internationale de services commerciaux. Une majorité d'électeurs hongkongais souhaite également préserver un minimum d'équilibre des pouvoirs en élisant des candidats démocrates au Legco. L'existence du mouvement pour la dé- mocratie n'est donc pas menacée dans un futur prévisible, malgré ses problèmes financiers et le traitement qui lui est réservé par les médias.

Les perspectives à court terme ne sont pourtant pas réjouissantes dans la mesure où le suffrage universel ne sera vraisemblablement pas instauré en 2012. Les autorités chinoises continueront parallèlement leurs efforts pour limiter l'ampleur du mouvement et soutenir le gouvernement de Donald Tsang. En l'absence d'un évènement fédérateur comme les protestations du $1^{\text {er }}$ juillet 2003, les groupes démocrates ne peuvent pas espérer une avancée significative aux élections locales de 2007 et aux élections législatives de 2008.

Une meilleure coordination entre les différents groupes qui constituent le mouvement démocratique et une contribution accrue aux grandes questions politiques comme l'éducation et la couverture sociale sont les prochains grands défis à relever pour les partis démocrates. Ils seront jugés par les électeurs et les médias sur leurs réalisations dans ces domaines. Si, jusqu'à présent, leur bilan est un peu faible, un pessimisme excessif n'est pas non plus de mise.

\section{- Traduit par Elsa Chalaux}

